\title{
Efecto de la zeolita nanoestructurada con ácido lipoico sobre los parámetros productivos y rendimiento de la canal en el pollo de engorda
}

\section{Effect of nanostructured zeolite with lipoic acid on performance and carcass yield in broiler}

\author{
Ofelia Mora Izaguirre ${ }^{a^{*}}$ \\ Katrin Quester ${ }^{\mathrm{b}}$ \\ Vitalii Petranovski ${ }^{b}$ \\ Laura González Dávalos ${ }^{\mathrm{a}}$ \\ Enrique Piña Garza ${ }^{c}$ \\ Armando Shimada Miyasaka ${ }^{\mathrm{a}}$ \\ Rafael Vazquez-Duhalt ${ }^{\mathrm{b}}$
}

${ }^{a}$ Laboratorio de Rumiología y Metabolismo Nutricional (RuMeN), Secretaría de Posgrado, Facultad de Estudios Superiores-Cuautitlán, UNAM, Tel 01442 2381032. Blvd. Juriquilla 3001 Col. Juriquilla, 76230, Querétaro, Qro. México.

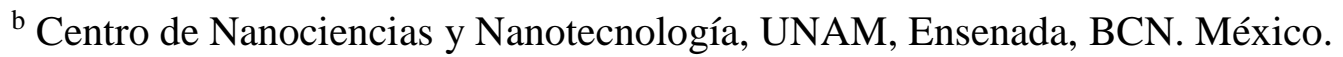

${ }^{\text {c }}$ Departamento de Bioquímica, Facultad de Medicina, UNAM. Ciudad de México. México.

Autor de correspondencia: ofemora2001@yahoo.com.mx

\section{- Resumen:}

Los antioxidantes se emplean rutinariamente como aditivos alimenticios en la producción de pollos de engorda. Entre ellos destaca el ácido alfa lipoico (AAL), ya que su adición en la dieta (40 $\mathrm{mg} \mathrm{kg}^{-1}$ ) mejora la ganancia de peso y la conversión alimenticia. Dado el alto precio del AAL, el objetivo de este trabajo fue estudiar el uso al combinarlo con nanoestructuras, 
las cuales pudieran conferirle ventajas de asimilación y por consiguiente, abatir costos de producción por concepto de alimentación. Para ello el AAL se inmovilizó con zeolita nanoestructurada tipo mordenita (Zeo) y se compararon sus efectos con las del AAL libre. La capacidad antioxidante del complejo (Zeo-AAL) es menor que la del compuesto libre, aun así, Zeo-AAL muestra una capacidad antioxidante importante. El AAL se libera mejor del complejo Zeo-AAL si éste se coloca en un ambiente simulando las condiciones del tubo digestivo, en comparación con su liberación en un ambiente sanguíneo. En la dieta de los pollos se evaluó el efecto del AAL libre (40 $\left.\mathrm{mg} \mathrm{kg}^{-1}\right)$ comparativamente con los del Zeo-AAL (0, 10 y $40 \mathrm{mg} \mathrm{kg}^{-1}$ ), sobre las variables productivas y el rendimiento de la canal. El AAL nanoestructurado con Zeo no representó ninguna ventaja sobre el AAL libre $(P>0.10)$, por lo que deberán de seguirse buscando estrategias con otras nanopartículas o compuestos nanoestructurados que permitan en un futuro bajar el costo en el uso del AAL sin disminuir sus beneficios probados.

- Palabras clave: Acido lipoico, Zeolita nanoestructurada, Pollos de engorda, Parámetros productivos.

\section{- Abstract:}

Antioxidants are routinely used as feed additives for broiler production. Among them alpha lipoic acid (ALA) in worth noting as its inclusion (40 $\left.\mathrm{mg} \mathrm{kg}^{1}\right)$ increases body weight gain and improves the feed conversion ratio. However, due to its elevated cost, the objective of this work was to study the use when combining it with nanostructures, which could confer its reported advantages and, consequently, lower production costs for food. Thus, the ALA was immobilized onto nanostructured zeolite type mordenite (Zeo) and its effect were compared with those of the free ALA. The antioxidant capability of the ZEO-ALA complex was lower than that of free ALA; however, it still showed an important antioxidant function. ALA is faster released from the complex when it is placed in an environment that simulates the conditions of the digestive tract than in a blood environment. Free ALA (40 mg kg- ${ }^{1}$ ) was compared to two concentrations of ZEO-ALA (equivalent to 10 and $40 \mathrm{mg} \mathrm{kg}^{-}{ }^{1}$ of ALA) on productive and carcass parameters. There were no statistically significant advantages of ZEO-ALA over ALA so more alternatives for lowering the cost of the latter should be looked upon.

- Key words: Lipoic acid, Nanostructurated zeolite, Broilers, Productive parameters.

Recibido el 08/11/2016.

Aceptado el 27/08/2017. 


\section{VIntroducción}

El ácido alfa lipoico (AAL), es un derivado del ácido octanóico. Además de su papel antioxidante, se sabe que participa como cofactor de dos complejos enzimáticos importantes en el ciclo de Krebs, la piruvato deshidrogenasa y la alfa-cetoglutarato deshidrogenasa ${ }^{(1)}$. Las células eucariotas son capaces de obtener el ácido lipoico de dos fuentes: a) exógena, proveniente de la dieta o de la síntesis de las bacterias intestinales y b) endógena, que proviene de la síntesis de novo a partir de ácido octanóico. Para el primer caso, en el intestino delgado el enterocito puede absorberlo a través de los receptores para ácidos grasos, tales como la proteína transportadora de ácidos grasos 4 (FATP4). Una vez dentro del enterocito es transportado al retículo endoplásmico por la misma FATP4 o por la proteína de unión a los ácidos grasos intestinal o del hígado (FABP2-1) e incorporarlo a los prequilomicrones para después pasar al aparato de Golgi y finalmente salir de la célula en los quilomicrones ${ }^{(2)}$.

El AAL ha sido usado en animales, tanto de laboratorio como de producción (pollo de engorda, codornices y cerdos), donde se ha observado que su principal efecto es una mejora en la ganancia de peso y la disminución en problemas asociados con el estrés calórico o el síndrome ascítico ${ }^{(3-7)}$. En nuestro grupo de trabajo se han observado efectos positivos sobre la ganancia diaria de peso (GDP) y la conversión alimenticia (CA) en pollos de engorda, por el uso de AAL a la dosis de $40 \mathrm{mg} \mathrm{kg}^{-1}$ en la dieta, sin embargo el costo de inclusión es alto $^{(5,8)}$. El objetivo de este trabajo es bajar el costo de la inclusión del AAL en la dieta, y por ende abaratar y propagar su uso en granjas comerciales. Para ello se exploró la posibilidad de usarlo inmovilizado en un material nanoestructurado como la zeolita (Zeo).

La zeolita se ha utilizado ampliamente como catalizador y adsorbente en la industria química. Recientemente se han ampliado sus usos en las áreas biológicas y en la protección del medio ambiente $^{(9)}$, todo ello sobre la base de sus propiedades, estabilidad en ambientes biológicos y comprobada inocuidad en organismos vivos ${ }^{(10)}$. Las zeolitas son materiales nanoestructurados consistentes en aluminosilicatos cristalinos que forman canales y cavidades de dimensiones nanométricas. Cuando están deshidratadas, las zeolitas tienen propiedades adsorbentes originadas por la presencia de canales micro- y nano-métricos, así como por la presencia de cationes que compensan la excesiva carga negativa del esqueleto aluminosílico. Los centros de adsorción en las zeolitas interaccionan con las moléculas adsorbidas por medio de fuerzas electrostáticas. A éstas se suman las interacciones provenientes de las fuerzas de 
dispersión, conduciendo de esa forma la existencia de una considerable energía de adsorción en los substratos zeolíticos ${ }^{(10)}$.

Las estructuras bien definidas de las zeolitas, combinadas con su naturaleza hidrofílica/hidrofóbica, las hacen tamices moleculares y hospedadores para diversas moléculas (orgánicas e inorgánicas) ${ }^{(11,12)}$.

Basándose en las propiedades fisicoquímicas mencionadas para las zeolitas, la hipótesis fue que al inmovilizar el AAL en la Zeo empleada, se garantizaría una liberación continua y constante de pequeñas cantidades del AAL, suficientes para reemplazar ventajosamente una carga única y alta de AAL. Al usar menos AAL inmovilizado en la Zeo, sin menguar sus ventajas productivas anotadas, disminuiría el costo de la dieta.

Por lo tanto, se midió el efecto del AAL inmovilizado en zeolita nanoestructurada (Zeo-AAL) a las dosis de 10 y $40 \mathrm{mg} \mathrm{kg}^{-1}$ y se comparó con el obtenido a la dosis de $40 \mathrm{mg} \mathrm{kg}^{-1}$ de AAL libre; ambos incorporados en la dieta de pollos de engorda, para comparar su efecto en las variables productivas y el rendimiento de la canal.

\section{Material y métodos}

\section{- Inmovilización del ácido lipoico en zeolita •}

Se empleó zeolita (Zeo) del tipo mordenita (MOR), proveniente de los depósitos naturales de Puebla, México. Diez kilos de ésta se deshidrataron en etanol anhidro durante $24 \mathrm{~h}$. El etanol se removió y se lavó nuevamente con etanol anhidro por 12 h más. El etanol se decantó y 100 g de ácido lipoico disueltos en etanol se adicionaron a la zeolita e incubados bajo agitación por $12 \mathrm{~h}$. Finalmente, el etanol se evaporó y se secó la Zeo con el AAL inmovilizado (Zeo-AAL).

\section{-Actividad antioxidante}


Se evaluó el efecto antioxidante del AAL libre o Zeo-AAL. La actividad antioxidante del AAL se obtuvo con el método de Marklund y Marklund ${ }^{(13)}$ modificado, midiendo la inhibición producida por el AAL en la oxidación espontánea del pirogalol en presencia del oxígeno atmosférico. Para ello, se utilizó una solución $2 \mathrm{mM}$ de pirogalol disuelta en buffer Tris-HCl a pH 8.2 y con 1 mM de EDTA. Antes de la medición, el buffer se oxigenó con aire. El $100 \%$ de oxidación se midió en ausencia de AAL, y la capacidad de inhibición de la oxidación se ensayó con la adición de diferentes cantidades de AAL o Zeo-AAL (0.0, 6.05, 12.10, 18.15 у 24.2 $\mathrm{mM})$. La solución stock del AAL libre o el inmovilizado se preparó usando $250 \mathrm{mg}$ de ácido lipoico, o 25 g Zeo-AAL, respectivamente, en $10 \mathrm{ml}$ etanol-buffer (1:1). La velocidad de oxidación se monitoreó a $420 \mathrm{~nm}$ en un espectrofotómetro UV/VIS de (Lambda 25, Perkin Elmer, USA).

\section{- Liberación del ácido lipoico •}

La liberación del AAL una vez incorporado a la Zeo se evaluó bajo dos condiciones:

1) Simulando el ambiente estomacal; para ello a $1 \mathrm{~g}$ de Zeo-AAL se adicionaron a $20 \mathrm{ml}$ de una solución de $\mathrm{HCl}$ a pH 2 y se mantuvo en agitación. Una alícuota de $1 \mathrm{ml}$ se retiró cada 5 min, centrifugados a 13,000 rpm y el sobrenadante se usó para estimar el AAL libre por espectrofotometría a $330 \mathrm{~nm}$ (Lambda 25, Perkin Elmer, EUA). La liberación se monitoreó hasta que no hubo más AAL liberado.

2) Simulando el ambiente sanguíneo, la Zeo-AAL se disolvió en $20 \mathrm{ml}$ de medio Eagle modificado por Dulbecco (Dulbecco’s modified Eagles Medium, DMEM, Sigma-Aldrich, EUA). La liberación del AAL se monitoreó de la manera descrita. Todos los ensayos se realizaron por triplicado.

\section{- Animales}


El trabajo se desarrolló en la granja avícola experimental de Integración y Desarrollo Agropecuario SA de CV, localizada en el Municipio de Charo, Estado de Michoacán, México; a una altura de 1,940 msnm. Se utilizaron 1,176 pollitos machos de 1 día de edad de la estirpe Cobb 500, los cuales se mantuvieron en producción hasta los 42 días de edad.

\section{- Tratamientos •}

Los animales se distribuyeron completamente al azar en cuatro tratamientos con siete repeticiones de 42 aves cada una, para determinar si el compuesto diseñado Zeo-AAL tiene un mejor efecto que el AAL libre. Los tratamientos utilizados se explican en el Cuadro 1.

Cuadro 1: Tratamientos aplicados

\begin{tabular}{lccc}
\hline Tratamiento & $\begin{array}{c}\text { AAL nanoestructurado } \\
\text { con zeolita }(\mathbf{m g})^{*}\end{array}$ & $\begin{array}{c}\text { AAL no nanoestructurado } \\
\text { con zeolita }(\mathbf{m g})\end{array}$ & $\begin{array}{c}\text { Zeolita no } \\
\text { nanoestructurada } \\
\left(\mathbf{k g ~ t}^{-1}\right)\end{array}$ \\
\hline Ze0-AAL40 & 40 & 0 & 0 \\
Zeo-AAL10 & 10 & 0 & 3 \\
Ze00 & 0 & 0 & 4 \\
AAL40 & 0 & 40 & 4 \\
\hline
\end{tabular}

* El ácido lipoico fue nanoestructurado con zeolita en una proporción de 40 y $10 \mathrm{mg}$ de lipoato/kg de zeolita.

\section{- Alimentación}


El alimento se preparó en forma de harina, una para los primeros 21 días de vida de las aves, otro para los siguientes 21 días (Cuadro 2), y se ofreció a libre acceso, al igual que el agua. El alimento se formuló y proporcionó por la empresa Integración y Desarrollo Agropecuario SA de CV. Cada semana de edad de las aves, se tomó directamente de los comederos una muestra de alimento de aproximadamente 50 g por cada tratamiento (para un total de 24 muestras), mismas que se colocaron en bolsas de plástico, identificadas por fecha y tratamiento, y se conservaron en refrigeración para su posterior análisis proximal y de contenido de AAL (Cuadro 2). 
Cuadro 2: Ingredientes y composición nutrimental de las dietas utilizadas durante los periodos de crecimiento (1-21 días) y finalización (22-42 días)

\begin{tabular}{|c|c|c|c|c|}
\hline \multirow[b]{3}{*}{ Premezcla Experimental } & \multicolumn{2}{|c|}{$0-21$ dias } & \multicolumn{2}{|c|}{ 22-42 dias } \\
\hline & Iniciador & $\mathrm{Kg}$ & Finalizador & $\mathrm{Kg}$ \\
\hline & 4 & 1.12 & 4 & 4.48 \\
\hline Sorgo & 554.55 & 155.27 & 612.48 & 685.9776 \\
\hline Pasta de soya $46 \%$ & 354 & 99.12 & 277 & 310.24 \\
\hline Aceite de soya & 35 & 9.8 & 58 & 64.96 \\
\hline Fosfato monodicalcico 21/18 & 18.7 & 5.236 & 14.1 & 15.792 \\
\hline Carbonato de calcio $38 \%$ & 14.8 & 4.144 & 13.4 & 15.008 \\
\hline Sal refinada iodada & 3.3 & 0.924 & 2.7 & 3.024 \\
\hline MHA $84 \%$ & 4.48 & 1.25 & 3.65 & 4.088 \\
\hline L-lisina $\mathrm{HCl}$ & 3.2 & 0.896 & 2.8 & 3.136 \\
\hline Bicarbonato de sodio & 2.2 & 0.616 & 2 & 2.24 \\
\hline Prem. Vit+Min+Colina & 4 & 1.12 & 4 & 4.48 \\
\hline Enramicina & 0.125 & 0.04 & 0.125 & 0.14 \\
\hline Nicarbazina & 0.5 & 0.14 & 0 & 0 \\
\hline Treonina & 1 & 0.28 & 0.8 & 0.896 \\
\hline Antioxidante & 0.15 & 0.042 & 0.15 & 0.168 \\
\hline Pigmento $20 \mathrm{~g} / \mathrm{kg}$ & 0 & 0 & 4 & 4.48 \\
\hline Carophil rojo $1 \%$ & 0 & 0 & 0.25 & 0.28 \\
\hline Salinomicina & 0 & 0 & 0.55 & 0.616 \\
\hline TOTAL & 1000 & 280 & 1000 & 1120 \\
\hline \multicolumn{5}{|c|}{ Composición estimada de las dietas } \\
\hline Proteína cruda, \% & 22 & & 18.5 & \\
\hline EM aves, Kcal/kg & 3025 & & 3225 & \\
\hline Grasa, \% & 5.0 & & 7.3 & \\
\hline Lisina total, \% & 1.42 & & 1.16 & \\
\hline Metionina+cistina, \% & 1.04 & & 0.87 & \\
\hline Treonina total, \% & 0.90 & & 0.74 & \\
\hline Triptofano, \% & 0.28 & & 0.23 & \\
\hline Calcio total, $\%$ & 1.09 & & 0.88 & \\
\hline Fósforo disponible, \% & 0.50 & & 0.40 & \\
\hline Sodio, $\%$ & 0.20 & & 0.178 & \\
\hline
\end{tabular}


Se utilizó una caseta de $10 \mathrm{~m}$ de ancho x 40 m de largo con techo de lámina de asbesto, con una capacidad de 28 pisos, cada uno de ellos con medidas de 2.5 x $4.0 \mathrm{~m}$, los cuales tienen dos comederos de tolva con un diámetro de $45 \mathrm{~cm}$ y con capacidad de $12 \mathrm{~kg}$ cada uno, así como un bebedero automático redondo tipo Plasson. A la llegada de los pollitos a la granja experimental, se pesaron en un solo grupo y se distribuyeron al azar en los 28 pisos mencionados.

El programa de manejo y sanitario fue similar para todos los tratamientos y sus respectivas réplicas; en la planta incubadora se aplicó la vacuna contra Marek y en la granja experimental, dos vacunas contra la enfermedad de Newcastle por vía ocular y oral (cepa La Sota) a los 8 y 25 días de edad, respectivamente. Se utilizó el fotoperiodo de luz natural.

Al inicio de la prueba se colocó un termómetro digital (RhT10, Extech Instruments, EUA), por dentro de la caseta a la altura de las aves, el cual fue programado para registrar la temperatura $\left({ }^{\circ} \mathrm{C}\right)$, la humedad $(\%)$ y el punto de rocío $\left({ }^{\circ} \mathrm{C}\right)$ ambiental, durante cada hora por el tiempo de estudio. A $\operatorname{los} 7,14,21,28,35$ y 42 días se tomaron las variables que se describen:

a) Peso de las aves: Se pesaron la totalidad de los pollos semanalmente en cada réplica y se calculó el peso individual promedio, acorde con el número de aves vivas al momento del pesaje.

b) Consumo voluntario de alimento: se pesó el alimento ofrecido al inicio de cada semana de edad de las aves, se recolectó y pesó el residual de cada réplica al final de la semana de edad de las aves. Se calculó el consumo individual promedio según el número de aves vivas al final de la semana.

c) Conversión alimenticia comercial: con los datos del promedio del peso corporal semanal y el consumo voluntario de alimento, se obtuvo la conversión alimenticia por semana y acumulada, la cual quedó bajo la siguiente fórmula: promedio del consumo voluntario de alimento entre la ganancia de peso corporal. La ganancia de peso corporal se obtuvo del peso corporal promedio, menos el peso corporal a la llegada del pollito, el cual fue de $47 \mathrm{~g}$.

d) Conversión alimenticia corregida por la mortalidad: este parámetro se obtuvo con el consumo de alimento acumulado (kilos), entre los kilos de peso corporal producidos; tomando en cuenta también los pesos de la mortalidad en la semana de estudio.

e) Mortalidad general: a las aves muertas se les realizó la necropsia para determinar la causa de muerte y se anotaron en la bitácora de cada réplica con la fecha de dicho acontecimiento; de esta forma se obtuvo el número de aves muertas por período y tratamiento. El porcentaje de mortalidad general acumulada y la ocasionada por el síndrome ascítico, se obtuvieron 
dividiendo el número de aves muertas, por 100, entre el número de aves que se iniciaron en el trabajo (42 aves).

Al término del experimento productivo (42 días) se pesaron y sacrificaron 84 machos ( 3 por repetición, 28 por tratamiento), para registrar individualmente: el peso corporal vivo, el peso de la canal (sin sangre, sin plumas, sin vísceras y sin patas), el peso de la grasa abdominal, el peso de la pechuga con hueso y el peso de muslos y piernas con hueso.

\section{- Análisis estadísticos}

Los experimentos de actividad antioxidante y liberación de AAL se llevaron a cabo en tres replicas independientes y se reporta el valor medio con la desviación estándar. Los resultados de los parámetros productivos y la mortalidad se analizaron en un diseño completamente al azar con mediciones repetidas, empleando las semanas de edad como variable del tiempo; cuando existieron diferencias significativas $(P<0.05)$, entre tratamientos, se realizó la comparación de medias por la prueba de diferencias mínimo cuadráticas. El rendimiento en canal, se analizó mediante un modelo completamente al azar de una sola vía ${ }^{(14)}$.

\section{Resultados}

La actividad antioxidante de las muestras de AAL libre y el nanoestructurado se indican la Figura 1A. Como se observa, la capacidad antioxidante del Zeo-AAL disminuye en comparación con el libre y éste muestra un comportamiento lineal.

La liberación del AAL estructurado a la Zeo (preparada a una concentración de $10 \mathrm{~g} \mathrm{~kg}^{-1}$ ) fue mucho mayor en el medio ácido, simulando las condiciones estomacales (Figura 1B), que en el medio sintético simulando el suero sanguíneo (Figura 1C). Lo anterior indica que una proporción importante del AAL inmovilizado se desprende de su nanoestructura al encontrarse el tubo digestivo de los pollos. 
Figura 1: Capacidad antioxidante del Zeo-AAL y AAL libre (A) y curvas de desorción en medio ácido (B) y en medio sintético $(\mathrm{C})$
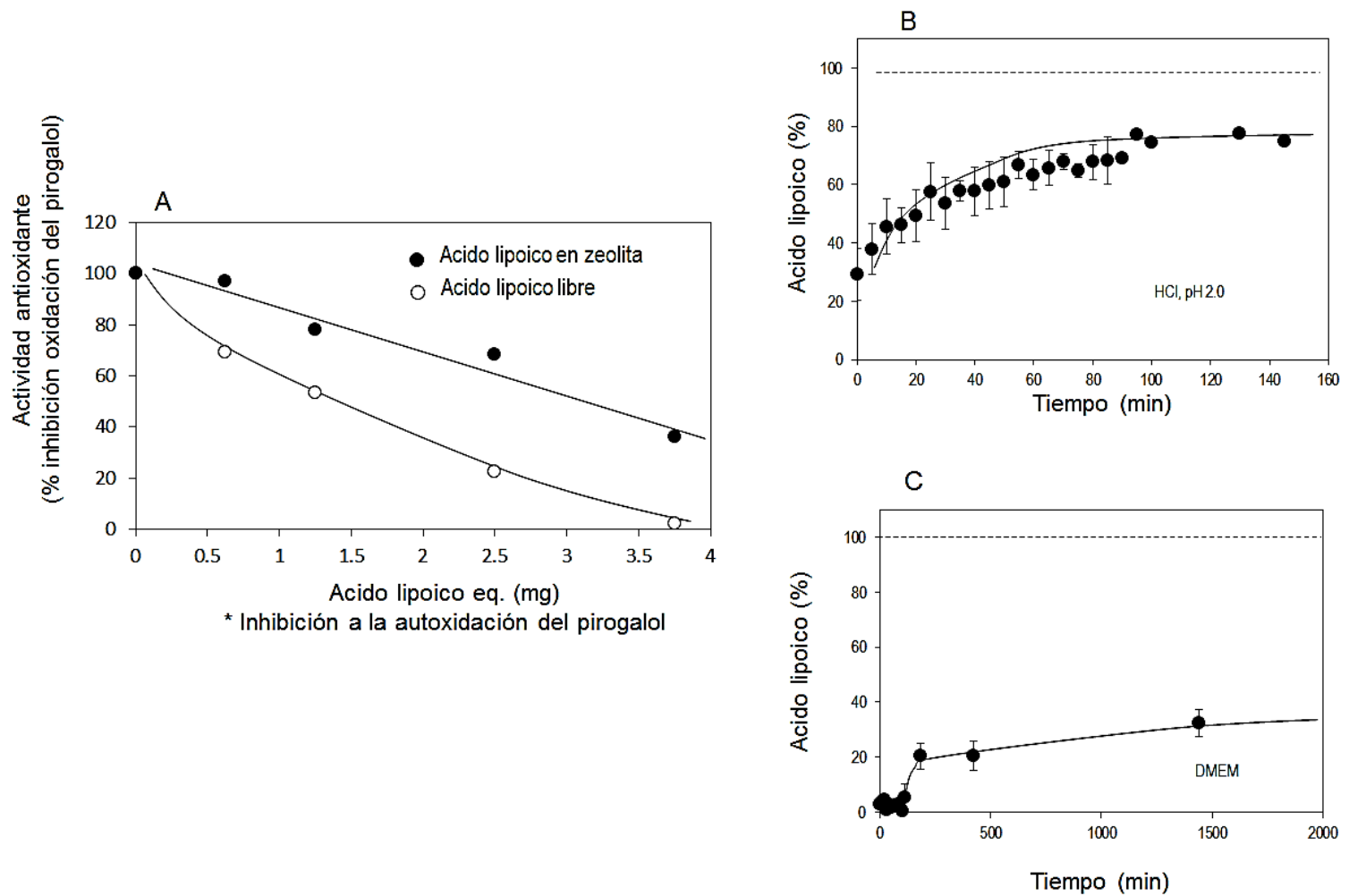

Los resultados del peso vivo de los pollos se muestran en el Cuadro 3, en donde se observa que las diferencias $(P<0.01)$ entre los tratamientos evaluados se manifiestan a partir de los 35 días de edad. Cuando los tratamientos Zeo-AAL10 y AAL40 fueron los que mostraron los mejores pesos vivos. Sin embargo, sólo el tratamiento AAL40 fue en el que se observó un peso mayor al final de la prueba (42 días de edad). En el consumo de alimento, también se observaron diferencias $(P<0.01)$ entre los tratamientos evaluados a partir de los 35 días de edad, siendo el tratamiento AAL40, el que mostró los mayores consumos de alimento al final de la prueba. 
Cuadro 3: Efecto de la zeolita nanoestructurada con ácido lipoico sobre el peso vivo y sobre el consumo de alimento acumulado en el pollo de engorda a través del tiempo*

\begin{tabular}{|c|c|c|c|c|c|c|c|c|c|c|c|c|}
\hline \multirow{3}{*}{ Tratamiento } & \multicolumn{12}{|c|}{ Días } \\
\hline & \multicolumn{2}{|l|}{7} & \multicolumn{2}{|c|}{14} & \multicolumn{2}{|c|}{21} & \multicolumn{2}{|c|}{28} & \multicolumn{2}{|c|}{35} & \multicolumn{2}{|c|}{42} \\
\hline & Peso vivo & CAA & $\begin{array}{l}\text { Peso } \\
\text { vivo }\end{array}$ & CAA & $\begin{array}{l}\text { Peso } \\
\text { vivo }\end{array}$ & CAA & $\begin{array}{l}\text { Peso } \\
\text { vivo }\end{array}$ & CAA & $\begin{array}{l}\text { Peso } \\
\text { vivo }\end{array}$ & CAA & $\begin{array}{l}\text { Peso } \\
\text { vivo }\end{array}$ & CAA \\
\hline Zeo-AAL40 & 0.131 & 0.136 & 0.358 & 0.494 & 0.793 & 1.174 & 1.288 & 2.041 & $1.841^{\mathrm{ab}}$ & $3.067 \mathrm{ab}$ & $2.529^{b}$ & $4.304^{b}$ \\
\hline Zeo-AAL10 & 0.135 & 0.133 & 0.358 & 0.495 & 0.801 & 1.176 & 1.300 & 2.032 & $1.865^{\mathrm{a}}$ & $3.046 \mathrm{ab}$ & $2.553^{\mathrm{ab}}$ & $4.299^{b}$ \\
\hline Zeo0 & 0.130 & 0.132 & 0.359 & 0.501 & 0.802 & 1.175 & 1.294 & 2.026 & $1.816^{\mathrm{b}}$ & $3.025^{b}$ & $2.530^{\mathrm{b}}$ & $4.265^{b}$ \\
\hline AAL40 & 0.133 & 0.133 & 0.360 & 0.498 & 0.799 & 1.170 & 1.306 & 2.050 & $1.860^{\mathrm{a}}$ & $3.081^{\mathrm{a}}$ & $2.585^{a}$ & $4.363^{a}$ \\
\hline EEM & 0.001 & 0.001 & 0.003 & 0.002 & 0.003 & 0.003 & 0.004 & 0.004 & 0.006 & 0.008 & 0.006 & 0.011 \\
\hline
\end{tabular}

Los valores de la conversión comercial y la conversión corregida por mortalidad (Cuadro 4) no se vieron afectadas $(P>0.05)$ entre los tratamientos evaluados, durante el desarrollo de la prueba, al igual que los porcentajes de la mortalidad general (Cuadro 5) y de la ocasionada por el síndrome ascítico (Cuadro 6).

Cuadro 4: Efecto de la zeolita nanoestructurada con ácido lipoico sobre la conversión de alimento acumulada y ajustada a la mortalidad en el pollo de engorda a través del tiempo

\begin{tabular}{|c|c|c|c|c|c|c|c|c|c|c|c|c|}
\hline \multirow{3}{*}{ Tratamiento } & \multicolumn{11}{|c|}{ Días } & \\
\hline & \multicolumn{2}{|c|}{7} & \multicolumn{2}{|c|}{14} & \multicolumn{2}{|c|}{21} & \multicolumn{2}{|c|}{28} & \multicolumn{2}{|c|}{35} & \multicolumn{2}{|c|}{42} \\
\hline & CAA & CAAM & CAA & CAAM & CAA & CAAM & CAA & CAAM & CAA & CAAM & CAA & CAAM \\
\hline Zeo-AAL40 & 1.631 & 1.631 & 1.595 & 1.588 & 1.575 & 1.571 & 1.645 & 1.642 & 1.710 & 1.670 & 1.734 & 1.691 \\
\hline Zeo-AAL10 & 1.512 & 1.512 & 1.599 & 1.597 & 1.561 & 1.549 & 1.622 & 1.616 & 1.676 & 1.663 & 1.715 & 1.643 \\
\hline Zeo0 & 1.601 & 1.601 & 1.607 & 1.607 & 1.556 & 1.556 & 1.624 & 1.624 & 1.710 & 1.698 & 1.718 & 1.681 \\
\hline AAL40 & 1.546 & 1.546 & 1.596 & 1.589 & 1.558 & 1.554 & 1.628 & 1.618 & 1.700 & 1.668 & 1.719 & 1.697 \\
\hline EEM & 0.029 & 0.029 & 0.016 & 0.015 & 0.007 & 0.006 & 0.005 & 0.005 & 0.006 & 0.007 & 0.004 & 0.010 \\
\hline
\end{tabular}

$\mathrm{CAA}=$ Conversión de alimento acumulada $\mathrm{CAAM}=$ conversión alimento acumulada ajustada a la mortalidad; $\mathrm{EEM}=$ Error estándar de la media. 
Cuadro 5: Efecto de la zeolita nanoestructurada con ácido lipoico sobre el promedio de la mortalidad general en el pollo de engorda a través del tiempo

\begin{tabular}{|c|c|c|c|c|c|c|c|c|c|c|c|c|}
\hline \multirow{3}{*}{ Tratamiento } & \multicolumn{12}{|c|}{ Días } \\
\hline & \multicolumn{2}{|c|}{7} & \multicolumn{2}{|c|}{14} & \multicolumn{2}{|c|}{21} & \multicolumn{2}{|c|}{28} & \multicolumn{2}{|c|}{35} & \multicolumn{2}{|c|}{42} \\
\hline & MG & MGA & MG & MGA & MG & MGA & MG & MGA & MG & MGA & MG & MGA \\
\hline Zeo-AAL40 & 0 & 0 & 0.29 & 0.68 & 0.43 & 1.02 & 0.57 & 1.36 & 2.00 & 4.76 & 3.57 & 8.50 \\
\hline Zeo-AAL10 & 0 & 0 & 0.14 & 0.34 & 0.57 & 1.36 & 0.86 & 2.04 & 1.43 & 3.40 & 4.00 & 9.52 \\
\hline Zeo0 & 0 & 0 & 0.00 & 0.00 & 0.00 & 0.00 & 0.00 & 0.00 & 0.43 & 1.02 & 1.86 & 4.42 \\
\hline AAL40 & 0 & 0 & 0.29 & 0.68 & 0.43 & 1.02 & 0.86 & 2.04 & 2.00 & 4.76 & 2.86 & 6.80 \\
\hline EEM & 0 & 0 & 0.07 & 0.18 & 0.09 & 0.22 & 0.14 & 0.33 & 0.22 & 0.51 & 0.39 & 0.94 \\
\hline
\end{tabular}

MG= Mortalidad general; MGA= Mortalidad general acumulada; EEM= Error estándar de la media.

Cuadro 6: Efecto de la zeolita nanoestructurada con ácido lipoico sobre la mortalidad acumulada por síndrome ascítico y sobre el porcentaje acumulado de la mortalidad ocasionada por síndrome ascítico en el pollo de engorda a través del tiempo

\begin{tabular}{|c|c|c|c|c|c|c|c|c|c|c|c|c|}
\hline \multirow{3}{*}{ Tratamiento } & \multicolumn{12}{|c|}{ Días } \\
\hline & \multicolumn{2}{|c|}{7} & \multicolumn{2}{|c|}{14} & \multicolumn{2}{|c|}{21} & \multicolumn{2}{|c|}{28} & \multicolumn{2}{|c|}{35} & \multicolumn{2}{|c|}{42} \\
\hline & MSA & $\begin{array}{c}\text { MSA } \\
(\%)\end{array}$ & MSA & $\begin{array}{c}\text { MSA } \\
(\%)\end{array}$ & MSA & $\begin{array}{c}\text { MSA } \\
(\%)\end{array}$ & MSA & $\begin{array}{c}\text { MSA } \\
(\%)\end{array}$ & MSA & $\begin{array}{c}\text { MSA } \\
(\%)\end{array}$ & MSA & $\begin{array}{c}\text { MSA } \\
(\%)\end{array}$ \\
\hline Zeo-AAL40 & 0 & 0 & 0 & 0 & 0.14 & 0.34 & 0.29 & 0.68 & 1.71 & 4.08 & 3.29 & 7.82 \\
\hline Zeo-AAL10 & 0 & 0 & 0 & 0 & 0.14 & 0.34 & 0.29 & 0.68 & 0.86 & 2.04 & 3.43 & 8.16 \\
\hline Zeo0 & 0 & 0 & 0 & 0 & 0.00 & 0.00 & 0.00 & 0.00 & 0.43 & 1.02 & 1.86 & 4.42 \\
\hline AAL40 & 0 & 0 & 0 & 0 & 0.14 & 0.34 & 0.43 & 1.02 & 1.57 & 3.74 & 2.43 & 5.78 \\
\hline EEM & 0 & 0 & 0 & 0 & 0.06 & 0.14 & 0.08 & 0.20 & 0.17 & 0.40 & 0.33 & 0.78 \\
\hline
\end{tabular}

$\mathrm{MSA}=$ Mortalidad acumulada por síndrome ascítico; EEM= Error estándar de la media.

Existieron diferencias $(P<0.01)$ en el rendimiento en la canal (Cuadro 7$)$ de los diferentes tratamientos. Los tratamientos Zeo-AAL10 y AAL40, fueron los que presentaron los mejores pesos corporales y mayores pesos de la canal. Expresados como porcentaje del peso vivo, sólo la pierna y muslo fueron diferentes para el tratamiento AAL40 $(P<0.02)$. 
Cuadro 7: Efecto de la zeolita nanoestructurada con ácido lipoico sobre el rendimiento en canal del pollo de engorda a los 42 días de edad

\begin{tabular}{lccccccccccc}
\hline Tratamiento & $\begin{array}{c}\text { Peso } \\
\text { corporal } \\
\text { vivo }(\mathbf{k g})\end{array}$ & $\begin{array}{c}\text { Peso } \\
\text { canal } \\
(\mathbf{k g})\end{array}$ & $\begin{array}{c}\text { Peso } \\
\text { vivo } \\
(\%)\end{array}$ & $\begin{array}{c}\text { Musloy } \\
\text { pierna } \\
(\mathbf{k g})\end{array}$ & $\begin{array}{c}\text { Peso } \\
\text { vivo } \\
(\%)\end{array}$ & $\begin{array}{c}\text { Pechuga } \\
(\mathbf{k g})\end{array}$ & $\begin{array}{c}\text { Peso } \\
\text { vivo } \\
(\%)\end{array}$ & $\begin{array}{c}\text { Alas } \\
(\mathbf{k g})\end{array}$ & $\begin{array}{c}\text { Peso } \\
\text { vivo } \\
(\%)\end{array}$ & $\begin{array}{c}\text { Grasa } \\
\text { abdominal } \\
(\mathbf{k g})\end{array}$ & $\begin{array}{c}\text { Peso } \\
\text { vivo } \\
(\%)\end{array}$ \\
\hline Zeo-AAL40 & $2.523^{\mathrm{b}}$ & $1.876^{\mathrm{y}}$ & 74.4 & $0.440^{\mathrm{b}}$ & $17.4^{\mathrm{b}}$ & 0.608 & 24.1 & 0.183 & 7.2 & 0.050 & 1.99 \\
Zeo-AAL10 & $2.565^{\mathrm{a}, \mathrm{b}}$ & $1.920^{\mathrm{x}, \mathrm{y}}$ & 74.9 & $0.443^{\mathrm{b}}$ & $17.3^{\mathrm{b}}$ & 0.616 & 24.0 & 0.185 & 7.2 & 0.049 & 1.91 \\
Ze00 & $2.52^{\mathrm{b}}$ & $1.878^{\mathrm{y}}$ & 74.3 & $0.445^{\mathrm{b}}$ & $17.6^{\mathrm{a}, \mathrm{b}}$ & 0.607 & 24.0 & 0.185 & 7.3 & 0.049 & 1.95 \\
AAL40 & $2.596^{\mathrm{a}}$ & $1.962^{\mathrm{x}}$ & 75.6 & $0.471^{\mathrm{a}}$ & $18.1^{\mathrm{a}}$ & 0.624 & 24.0 & 0.189 & 7.3 & 0.047 & 1.80 \\
EEM & 0.009 & 0.009 & 0.20 & 0.003 & 0.11 & 0.004 & 0.13 & 0.002 & 0.1 & 0.001 & 0.04 \\
\hline
\end{tabular}

${ }^{\mathrm{a}, \mathrm{b}}$ Diferentes literales en la misma columna indican diferencias $(P<0.01)$.

${ }^{\mathrm{x}, \mathrm{y}}$ Diferentes literales en la misma columna indican diferencias $(P<0.06)$.

w,z Diferentes literales en la misma columna indican diferencias $(P<0.02)$.

$\mathrm{EEM}=$ Error estándar de la media.

\section{| Discusión}

Los materiales sólidos porosos son de gran valor desde el punto de vista tecnológico, ya que pueden interactuar con átomos, iones y moléculas en toda su superficie. Sus aplicaciones más comunes comprenden el intercambio iónico, adsorción y catálisis; varias de estas aplicaciones se han beneficiado del alto grado de ordenamiento de materiales tales como las zeolitas $^{(15)}$. Los poros se clasifican en función de su tamaño y es la distribución de tamaños, forma y volumen ocupado por la porosidad lo que determina la utilidad del material para desempeñar una labor determinada. En función de la necesidad de contar con materiales con poros uniformes, surgieron los materiales nanoestructurados, que son aquellos sólidos en los que el control de su estructura se ejerce hasta la escala nanométrica (e incluso en algunos casos el control puede llegar a ser a nivel atómico) ${ }^{(15)}$. El hecho de que el tamaño del poro de estos materiales pueda ser "seleccionado" entre la escala 1.4 y $10 \mathrm{~nm}$, simplemente modificando las condiciones de síntesis ${ }^{(16)}$, los convierte en sólidos versátiles para distintas aplicaciones, tales como adsorbentes, catalizadores o soportes, o bien, como parte de reacciones de intercambio ${ }^{(17)}$.

Cuando se usaron diferentes proporciones de AAL, se observó que la preparación inmovilizada tiene una menor capacidad antioxidante que el compuesto libre. Sin embargo Zeo-AAL muestra 
una capacidad antioxidante significativa. La capacidad antioxidante es un poco menor en la preparación inmovilizada, lo cual puede ser originado por la falta de acceso del medio al grupo con capacidad antioxidante (-S-S-) de una proporción de las moléculas de AAL. Sin embargo, como se puede observar la capacidad antioxidante de la preparación inmovilizada es aún muy importante.

Respecto al uso de zeolita nanoestructurada (Zeo), Rahimi et al ${ }^{(12)}$ observaron que las proteínas plasmáticas se pueden adsorber en los canales de la Zeo. Ellos reportan que a la concentración del plasma de $100 \%$ existe una alta adsorción selectiva para la apolipoproteína C-III (APOC-III) y fibrinógeno en la nanoestructura de la Zeo. También se ha reportado el uso de los nanocanales de Zeo nanoestructurados con $\mathrm{ADN}^{(18)}$, para ser usados como una especie de agentes transfectantes con buenos resultados. Pascual ${ }^{(19)}$ reporta que los carotenoides de paprika, como ingrediente de interés alimentario, presentaron una mayor estabilidad contra la degradación cuando se embebieron en materiales nanoestructurados de Zeo, debido a que la adsorción de agua en la superficie se controló por mecanismos entrópicos. Sin embargo, en ninguno de estos reportes se menciona la cinética de liberación de los materiales biológicos de la Zeo y la suerte del material zeolitico después del tratamiento. En este sentido, los resultados obtenidos muestran un mejor comportamiento producto del AAL libre, lo que podría explicarse en función de su cinética de liberación.

Las nanoestructuras de origen biológico, se metabolizan y aprovechan para formar otras estructuras biológicas liberando sus cargas; en cambio, las nanoestructuras no biológicas como la Zeo no pueden ser metabolizadas ${ }^{(20)}$, por lo que serán excretadas, y era de esperarse que el AAL inmovilizado sería absorbido o retenido en el tracto gastrointestinal. En reportes previos de nuestro grupo de trabajo se observó que $40 \mathrm{mg} \mathrm{t}^{-1}$ de $\mathrm{AAL}^{(5,21)}$ mejora la ganancia de peso, algunas variables de la calidad de la canal y la mortalidad por síndrome ascítico; sin embargo en un análisis financiero respecto al uso de AAL en una granja comercial, Alvarez ${ }^{(8)}$ observó un aumento en los costos de alimentación por la adición del AAL, aunque se mejoró la rentabilidad con su uso.

\section{Conclusiones e implicaciones}

En este trabajo se diseñó una Zeo nanoestructurada conteniendo AAL inmovilizado para administrarse a pollos de engorda por vía oral, con el objetivo de ofrecer una dosis menor y abaratar los costos de suplementación del AAL. Sin embargo el uso del AAL en Zeo 
nanoestructurada no representó ninguna ventaja sobre el AAL libre, lo que significa que deberán de seguirse buscando alternativas con nanopartículas, u otros compuestos nanoestructurados, que permitan reducir la dosis de $40 \mathrm{mg} \mathrm{t}^{-1}$ de $\mathrm{AAL}$, sin disminuir los beneficios probados del AAL sobre las variables productivas en el pollo de engorda.

\section{Agradecimientos}

Este trabajo fue financiado por el Proyecto PAPIIT-UNAM IN200910. Se agradece la participación de Yumi Ghinis-Hozumi, Sergio Sigler Galván y Martín Barbosa Amezcua en el sacrificio de los animales.

\section{- Literatura Citada}

1. Nesbitt NM, Cicchillo RM, Lee KH, Grove TL, Booker SJ. Lipoic acid biosynthesis. In: Patel MS, Packer L editors. Lipoic acid, energy, production, antioxidant activity and health effects. Boca Ratón, Florida, USA: CRC Press; 2008:11-13.

2. Boudry G, David ES, Douard V, Monteiro IM, Le Huërou-Luron I, Ferraris RP. Role of intestinal transporters in neonatal nutrition: carbohydrates, proteins, lipids, minerals, and vitamins. J Pediatr Gastroenterol Nutr 2010;51(4):380-401.

3. Had M, Anjum F, Asghar A, Khan M, Yasin M, Shahid M, et al. Lipid stability and antioxidant profile of microsomal fraction of broiler meat enriched with r-LipoicaAcid and r-Tocopherol acetate. J Agric Food Chem 2011;59:7346-7352.

4. Berg E, Maddock K, Linville M. Creatine monohydrate supplemented in swine finishing diets and fresh pork quality: III. Evaluating the cumulative effect of creatine monohydrate and alpha-lipoic acid. J Anim Sci 2003;81(10):2469-2474.

5. Díaz-Cruz A, Serret M, Ramírez G, Ávila E, Guinzberg R, Piña E. Prophylactic action of lipoic acid on oxidative stress and growth performance in broilers at risk of developing ascites syndrome. Avian Pathol 2003;32(6):645-653. 
6. Halici M, Imik H, Koç M, Gümüş R. Effects of a-lipoic acid, vitamins E and C upon the heat stress in japanese quails. J Anim Physiol Anim Nutr 2012;96(3):408-415.

7. Hamano Y. Effects of dietary lipoic acid on plasma lipid, in vivo insulin sensitivity, metabolic response to corticosterone and in vitro lipolysis in broiler chickens. Br J Nutr 2006;95(6):1094-1101.

8. Alvarez AC. Empleo del ácido alfa lipoico para promover el crecimiento y mejorar los parámetros productivos del pollo de engorda en condiciones de granja comercial [tesis maestría]. Cuautitlán Izcalli, Estado de México: Universidad Nacional Autónoma de México; 2014.

9. Yu H, Lv Y, Ma K, Wang C, Xue Z, Zhao Y, et al. Synthesis of core-shell structured zeolite-A@mesoporous silica composites for butyraldehyde adsorption. J Colloid Interface Sci 2014;428:251. doi: 10.1016/jcis.2014.04.060.

10. Hernández MA, Rojas F, Corona L, Lara VH, Portillo R, Salgado MA, et al. Evaluación de la porosidad de zeolitas naturales por medio de curvas diferenciales de adsorción. Rev Int Contam Ambient 2005;21(2);71-81.

11. Farias-Piñeira T. Materiales compuestos zeolita-surfactante-fármaco con uso potencial en la industria farmacéutica [tesis doctorado]. La Habana, Cuba: Universidad de La Habana; 2010.

12. Rahimi M, Ng EP, Bakhtiari K, Vinciguerra M, Ali Ahmad H, Awala H, et al. Zeolite nanoparticles for selective sorption of plasma proteins. Sci Rep 2015;30:17259. doi:10.1038/srep17259.

13. Marklund S, Marklund G. Involvement of the superoxide anion radical in the autoxidation of pyrogallol and a convenient assay for superoxide dismutase. Eur $\mathrm{J}$ Biochem 1974;47:469-474.

14. SAS. SAS User's Guide. Cary NC, USA: SAS Inst. Inc. 2008.

15. Berenguer MA. Membranas zeolita/carbón, materiales nanoestructurados y coloides: síntesis y aplicaciones [tesis doctorado]. Alicante, España: Universidad de Alicante; 2005.

16. Ryoo R, Kim JM. Structural order in MCM-41 controlled by shifting silicate polymerization equilibrium. $\mathrm{J}$ Chem Soc Chem Commun 1995;7:711712.doi:10.1039/C39950000711.

17. Ciesla U, Schüth F. Ordered mesoporous materials. Microporous and mesoporous mater 1999;27:131-149. 
18. Lülf H, Bertucci A, Septiadi D, Corradini R, De Cola L. Multifunctional inorganic nanocontainers for DNA and drug delivery into living cells. Chem Eur J 2014;20:10900-10904. doi:10.1002/chem.201403232.

19. Pascual LA. Caracterización de nanoestructuras con diferente geometría: uso potencial en alimentos [tesis doctorado]. México, DF: Instituto Politécnico Nacional; 2010.

20. Nieto PA. Aplicaciones biomédicas de materiales mesoporosos de sílice y carbón [tesis doctorado]. Madrid, España: Universidad Complutense de Madrid; 2011.

21. Sigler-Galván S, Gómez-Rosales S, Alarcón-Rojo A, Angeles L, Piña E, ShimadaMiyasaka A, Mora-Izaguirre O. Efecto del ácido lipoico sobre parámetros productivos y calidad de la carne en el pollo de engorda. Rev Mex Cienc Pecu 2015;6(2):207-219. 